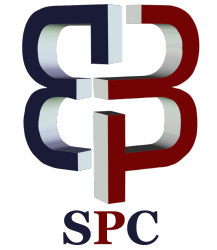

International Journal of Basic and Applied Sciences, 4 (4) (2015) 354-363

www.sciencepubco.com/index.php/IJBAS

(c) Science Publishing Corporation

doi: $10.14419 /$ ijbas.v4i4.5044

Research paper

\title{
LVP conditions at Mohamed V airport, Morocco: local characteristics and prediction using neural networks
}

\author{
Driss BARI ${ }^{1 *}$, Mohamed EL KHLIFI ${ }^{2}$ \\ ${ }^{1}$ National Center for Meteorological Research, National Directorate of Meteorology, Casablanca, Morocco \\ ${ }^{2}$ University Hassan II of Casablanca, Faculty of Sciences and Techniques Mohammedia \\ Laboratory of Mathematics, Cryptography and Mechanical, Morocco \\ *Corresponding author E-mail: bari.driss@gmail.com
}

Copyright (C2015 Driss BARI and Mohamed EL KHLIFI. This is an open access article distributed under the Creative Commons Attribution License, which permits unrestricted use, distribution, and reproduction in any medium, provided the original work is properly cited.

\begin{abstract}
Low visibility and/or ceiling conditions have a strong impact on airports' traffic and their prediction is still a challenge for meteorologists. In this paper, the local characteristics of Low Visibility Procedure (LVP) conditions are investigated and the artificial neural network (ANN) based on resilient backpropagation as supervised learning algorithm is used to predict such meteorological conditions at Mohamed V international airport, Casablanca, Morocco. This article aims to assess the ANN ability to provide accurate prediction of such events using the meteorological parameters from the Automated Weather Observation Station (AWOS) over the period from January 2009 to March 2015. First, LVP conditions were classified according to their classes ( fog LVP and no fog LVP ) and their sources (Runway Visual Range -RVR LVP-, Ceiling -HCB LVP- or both) for both runway end points (35R and 17L). It is found that most of LVP conditions are associated with fog and are often due to decreasing of RVR below $600 \mathrm{~m}$. Next, Eleven ANNs were developed to produce LVP prediction for consecutive hourly valid forecast times covering the night and early morning. The Multi-Layer Perceptron (MLP) architecture with one hidden layer is used in this study. Results show that ANNs are able to well predict the LVP conditions and are robust to errors in input parameters for a relative error below 10\%. Furthermore, it is found that the ANN's skill is less sensitive to LVP type being predicted.
\end{abstract}

Keywords: Low Visibility Procedure, Ceiling, Multi-Layer Perceptron, Neural Network, Resilient backpropagation.

\section{Introduction}

The occurrence of adverse ceiling and visibility conditions that restricted the flow of air traffic in major airport terminals is one of the main causes of aircraft delays and is crucial for air traffic safety and economic issues. For example, at Paris - Charles de Gaulle international airport, the landings and departures are reduced by a factor of 2 in foggy conditions (Bergot, 2007 [1]). On main international airports, the low visibility procedures (LVP) are applied for the purpose of ensuring safe operations. At Mohamed V international airport (GMMN), located in Casablanca, Morocco, LVP conditions, defined by Air Traffic Control authorities (ATC) according to the International Civil Aviation Organization (ICAO), correspond to visibility $<600 \mathrm{~m}$ or ceiling $<200$ feet (about $60 \mathrm{~m}$ ). 
The ceiling refers to the height above the ground or water of the base of the lowest layer of cloud below $6000 \mathrm{~m}$ $(20000 \mathrm{ft})$ covering more than half the sky (ICAO, 2012 [2]). Thus, providing ATC with meteorological forecasts which include the probability of visibility and/or ceiling conditions which may require LVP to be undertaken is fundamental to the successful aviation of the LVP phases. Unfortunately, current operational Numerical Weather Prediction (NWP) models are not able to provide detailed information due to the complex interaction between the physical processes during the life cycle of such LVP conditions.

The GMMN airport is equipped with a CAT-IIIA Instrument Landing System since 2008. This means that aircrafts can still land with a minimum runway visual range (RVR) of $200 \mathrm{~m}$ and a decision height lower than 100 feet (about $30 \mathrm{~m}$ ). The category IIIA requires that the pilots must be qualified and the aircraft should suitably be equipped. RVR is the range over which the pilot of an aircraft on the centre line of a runway can see the runway surface markings or the lights delineating the runway or identifying its centre line (ICAO, 2012 [2]). At GMMN, RVR is electronically measured by transmissometers at each end of the runway (35R and 17L) with another sensor at the centre point. The decision height is a specified altitude or height in the precision approach at which a missed approach must be initiated if the required visual reference to continue the approach has not been established (ICAO, 2012 [2]). The height of cloud base at GMMN airport is measured using ceilometer.

During the night of 21-22 January 2008, a dense fog event (visibility below $200 \mathrm{~m}$ ) which occurred at the Grand Casablanca region, and which lasted for more than 12 hours, obliged the diversion of 21 aircrafts which supposed to land at the airport Mohamed V, to other national airports. According to the World Meteorological Organization, fog is defined as a collection of suspended water droplets or ice crystals that are near the surface and lead to horizontal visibility below $1 \mathrm{~km}(\mathrm{WMO}, 1992$ [3]). Diverting flights to another airport results in considerable cost to airlines (e.g. extra fuel consumption and hotel accommodation for the passengers). This indicates the need to improve LVP conditions forecasting over the GMMN airport and provide the motivation to carry out this research.

Since the occurrence of the LVP conditions depends, among other factors, on the geographical location and season, improved knowledge of the circumstances, when LVP conditions occur or do not, based on local meteorological parameters is important as first step. This will serve as basis for further investigations of the factors leading to such meteorological conditions. This will in turn result in improved flight planning by the airlines operating of the airport.

In an attempt to overcome the aforementioned deficiencies, the present study integrates the measured meteorological parameters from Automated Weather Observation Station (AWOS) with high temporal resolution (each minute) and uses a non linear statistical technique. Given existing techniques available to operational forecasters, LVP conditions prediction may be improved by the development and application of Neural Networks (NN). In fact, Artificial Neural Networks (ANN) are computational methodologies capable of establishing non linear relationship between independent variables (predictors) and a dependent variable (predictand) through the experimentation of a multitude of situations (learning data set). Even if ANNs have existed for over 60 year, their first application in atmospheric science was in 1986 (Gardner and Dorling (1998) [4]; Hsieh and Tang (1998) [5]), and their application in fog forecasting is rather limited (Pasini et al. (2001) [6]; Fabian et al. (2006) [7]; Bremnes and Michaelides (2007) [8]). Marzaban et al. (2006) [9] compared neural network with linear and logistic regression in forecasting ceiling and low visibilities. They concluded that the ANN approach yields superior forecast quality. As far as the authors know, there is no studies which have dealt with LVP conditions using AWOS data.

The ANN-based low visibility forecasting models are developed for a specific valid time such as 0600UTC and 1200UTC in Marzaban et al. (2006) [9]. According to the authors, the sets of ANNs had not been developed for consecutive hourly valid times and from different lead times. Furthermore, most of these studies used the traditional backpropagation algorithm. So, the aim of this work is to develop a set of ANN-based LVP forecast models based on the resilient backpropagation algorithm (Günther and Fritsch (2010) [10]) as supervised learning algorithm. The developed models use the meteorological observations, from AWOS, as input data. To achieve this, the local characteristics of LVP conditions at the Mohamed V international airport are first determined using the available meteorological parameters over the period covering January 2009 to March 2015.

The paper is organized as follows. Theory of the resilient backpropagation, the dataset and the methodology used in this work are described in section 2. Next, the determination of the LVP-prone months and diurnal period and a description of general character of LVP conditions over the Mohamed V airport are detailed in section 3. In this section, the performance of the sets of ANNs developed is also established. Finally, some conclusions are 
suggested in section 4 .

\section{Materials and methods}

This section briefly surveys the theory of the supervised learning algorithm (resilient backpropagation) used in this work. The dataset used to construct the training and testing samples for Neural Networks and the methodology adopted in the development process are also described in this section.

\subsection{Supervised learning algorithm : resilient backpropagation}

Zhang et al. (1998) [11] mentioned that ANNs consist of collection of highly interconnected processing elements (neurons) that transforms a set of inputs to a set of outputs. The result of the transformation is determined by the characteristics of the neurons and weights associated with interconnections between them. The output of simplest Multi-Layer Perceptron (MLP) with one hidden layer, one output neuron and $n$ input variables, can be written as in [10] (2010) by Günther and Fritsch :

$Y=f\left(w_{0}+\sum_{k}^{H} w_{k} f\left(w_{0}^{k}+\sum_{i}^{n} w_{i}^{k} x_{i}\right)\right)$

where

$w_{0} \quad=$ the intercept of the output neuron

$w_{0}^{k} \quad=$ the intercept of the $\mathrm{k}^{\text {th }}$ hidden neuron

$w_{k} \quad=$ the synaptic weight corresponding to the synapse starting at the $\mathrm{k}^{\text {th }}$ hidden neuron and leading to the output neuron

$\left(w_{1}^{k}, \ldots, w_{n}^{k}\right) \quad=$ the vector of all synaptic weights corresponding to the synapses leading to the kth hidden neuron

$\left(x_{1}, \ldots, x_{n}\right) \quad=$ the vector of all covariates

$f \quad=$ the activation function is the sigmoid function defined by $f(x)=(1+\exp (-x))^{-1}$

$Y \quad=$ the output

$H \quad=$ the number of hidden neurons

The neural network training is an unconstrained non linear minimization problem in which the weights of a network are iteratively modified to minimize the distance $E$, called error function, between the desired $(Y)$ and actual output values $(O)$ :

$E=\frac{1}{2}\|Y-O\|^{2}$

The most popularly used training method is the backpropagation algorithm (BP) which is essentially a gradient steepest descent method. It is characterized by the usage of a given output that is compared to the predicted output and by the adaptation of all weights according to this comparison. This algorithm tries to minimize the error function by adding a learning rate $\eta$ to the weights going into the opposite direction of the gradient (Rumelhart and Clellend (1986) [12])

$w_{k}^{(t+1)}=w_{k}^{(t)}-\eta \frac{\partial E^{(t)}}{\partial w_{k}^{(t)}}$

where

$t \quad=$ the iteration

$E \quad=$ the error function

$w_{k}=$ the weights

Unlike the traditional backpropagation algorithm (Eq. 3 ), the resilient backpropagation (Eq. 4) algorithm (Anastasiadis et al. (2005) [13]) modifies the weights of neural network in order to find a local minimum of the error function with a separate learning rate $\eta_{k}^{(t)}$, which can be changed during the training process. This is done to solve the problem of using an over-all learning rate for the whole training process and so for the entire network. Additionally, instead of the partial derivatives magnitude only their sign is used to update the weights (Günther and Fritsch (2010) [10]):

$w_{k}^{(t+1)}=w_{k}^{(t)}-\eta_{k}^{(t)} \operatorname{sign}\left(\frac{\partial E^{(t)}}{\partial w_{k}^{(t)}}\right)$ 
In this study, the resilient backpropagation algorithm (Eq. 4) is used as supervised learning algorithm.

\subsection{Dataset and methodology}

At aerodromes with runways intended for category III instrument approach and landing operations, automated equipment are installed for measuring the following meteorological parameters:

- Surface wind

- Runway Visual Range

- Height of Cloud Base

- Air and dew-point temperatures

- Atmospheric pressure

The dataset used in this work is extracted from the AWOS archives and covers the period, from January 2009 to March 2015. The dataset contains hourly measurements of the following meteorological parameters: dry-bulb and dew-point temperatures $\left({ }^{\circ} \mathrm{C}\right), 2$ - and 10-min average wind speed $(\mathrm{m} / \mathrm{s}), 2$ - and 10-min average wind direction (degrees), 1- and 10-min maximum wind speed $(\mathrm{m} / \mathrm{s})$, mean sea level pressure $(\mathrm{hPa}), 10-\mathrm{min}$ and instantaneous minimum RVR at runway end points $35 \mathrm{R}$ and $17 \mathrm{~L}(\mathrm{~m})$, the height of the lowest cloud base $(\mathrm{m})$, relative humidity (\%) and atmospheric pressure at the station $(\mathrm{hPa})$. To complement this dataset, the hourly index $(0$ or 1$)$ is extracted from METAR whether fog occurs or does not.

First, the diurnal hourly range and the months which experience high frequency of LVP conditions are established using the monthly and hourly distribution of LVP occurrences over the studied period. These two characteristics (monthly and diurnal LVP period) are used during the development process of the ANN-based models. Besides, LVP are classified into two categories: Fog LVP and no-Fog LVP. The latter (no fog LVP) comprises the scenario when LVP conditions occur in association with any kind of precipitation or other hydrometeor (rain, shower, etc.). Furthermore, the sources (ceiling or RVR) leading to LVP to be undertaken are investigated by classifying the LVP conditions into the following categories:

1. RVR-LVP when RVR $<600 m$ and $\mathrm{HCB} \geq 60 m$

2. HCB-LVP when RVR $\geq 600 m$ and $\mathrm{HCB}<60 m$

3. BOTH-LVP when RVR $<600 m$ and $\mathrm{HCB}<60 \mathrm{~m}$

To select the appropriate subset of predictors for each NN-based developed model, the stepwise procedure with a forward selection is used in this work. The predictors' selection, from the 16 meteorological parameters, is done based on Akaike Information Criterion (AIC). This approach can ensure that the developed network architecture would be well adapted to the complexity of the phenomenon being predicted (Knerr et al. 1990 [14]).

Given that the input variables belongs to different ranges, scaling all inputs to the same range, using a normalization approach, prevents the MLP from placing unwarranted emphasis on those inputs with a larger mean magnitude. The target variable (output), in this study, is a binary indicator of either 'LVP' or 'no LVP'. The relative operating characteristic (ROC) is used here to determine the threshold value required to decide when to declare a LVP forecast. Thus, to maximize the MLP training efficiency, all input data were normalized based on formula (5):

$X=\frac{x-x_{\min }}{x_{\max }-x_{\min }}$

where $x_{\min }$ and $x_{\max }$ are the extremes values of the variable $x$ over the studied period.

Investigation in the dataset points out the existence of inequality between the numbers of LVP days and noLVP days during the LVP-prone months. Such inequality can cause problems during the development process of ANN-based models (Parikh et al., 1999 [15]). Fabian et al. (2007) [7] mentioned that, in cases where the class sizes 
are unequal, the NNs may converge on a solution by which the largest class is always forecast. In this study, the training and testing samples, extracted from the raw data, verify the condition of equality between the numbers of LVP days and no-LVP days. The study period was divided into two periods: from January 2009 to December 2012, and from January 2013 to March 2015. The data from the first period were used for training, while the second were employed for testing. The partition of the raw dataset into training and testing subsets was achieved by randomizing the inputs and their associated target values (Reusch and Alley, 2002 [16]). This is common practice during the MLP training to remove interseasonal variability. In an effort to minimize training times and avoid the risk of overparameterization, MLPs were trained with a single hidden layer.

In an operational point of view, the meteorological forecaster provides ATC, at any moment, with necessary information which may require LVP to be undertaken. To overcome the occasional missing values problem likely to occur in an operational context for a specific run, the used training and testing subsets had a complete set of observations and the eleven ANN-based models were developed to produce hourly fog prediction starting from hourly lead times from 1000UTC to 2000UTC. The valid forecast times for all ANNs cover the night and early morning (from 2100UTC to 0900TC) where the LVP conditions are common to occur at the GMMN airport (See section 3.1).

To assess the skill of all these models, the most common statistics are derived from the contingency table (Wilks, 1995 [17]). The statistics based on probability of detection $(P O D)$, false alarm rate $(F A R)$, and frequency bias index $(F B I)$ are computed:

$P O D=\frac{a}{a+c} \quad F A R=\frac{b}{a+b} \quad F B I=\frac{a+b}{a+c}$

where

$\mathrm{a}=$ the number of observed and predicted events

$\mathrm{b}=$ the number of unobserved and predicted events

$\mathrm{c} \quad=$ the number of observed and unpredicted events

The $P O D$ represents the likelihood that a LVP event would be forecast when it occurred. The $F A R$ refers to that proportion of LVP forecasts that fails to occur. The frequency bias index is a comparison of the average LVP forecast with the average observation. The perfect scores of $P O D, F A R$ and $F B I$ are respectively $100 \%, 0 \%$ and 1. Unbiased LVP forecasts exhibit $F B I=1$ indicating that the LVP was forecast the same number of times that it was observed.

\section{Results and discussion}

In this section, the LVP-prone months at the Mohamed V international airport are determined and the diurnal hourly range when the LVP conditions are very common to occur is outlined. Based on these two characteristics (LVPprone months and LVP diurnal period), the ANNs are developed to establish LVP forecasting aid for operational forecasters.

\subsection{Local characteristics of LVP conditions}

The monthly frequency of LVP conditions for both runway end points (35R and 17L) is shown in Figure 1 to illustrate more clearly its seasonal distribution (Fig. 1(a) for runway 35R and 1(b) for runway 17L). To determine the preferred time of day for LVP conditions' occurrence, the hourly distribution of LVP frequency is plotted in Fig. 1(c) for runway 35R and Fig. 1(d) for runway 17L. The frequency is defined as the total number of hourly LVP conditions per month divided by the total number of hourly LVP conditions for each runway end point. To deeply investigate the local characteristics of LVP conditions at GMMN airport, a distinction is made between the LVP type (Fog LVP and no-Fog LVP) and LVP source (HCB-LVP, RVR-LVP or Both).

There is a marked tendency toward a maximum likelihood of LVP occurrence during the cold season (from October to April) at GMMN airport, particularly in winter months. In fact, Fig. 1(a) and 1(b) point out that LVP conditions are often associated with foggy conditions except for February and November where no-Fog LVP type prevails. Early in autumn and late in summer, the LVP conditions are often due to no-Fog conditions. In relation to LVP' source, most of LVP conditions are due to decreasing of RVR below 600m (RVR-LVP). This scenario is found for both runway end points $(35 \mathrm{R}$ and $17 \mathrm{~L})$. A noteworthy feature is that LVP conditions are rare to occur 
when RVR does not exceed 600m and decision height is below 60m simultaneously (BOTH-LVP).

In terms of diurnal dependence (Fig. 1(c) and 1(d)), it is observed that LVP conditions occur mostly during the night time and early morning hours (from 2100UTC to 0900UTC). The LVP conditions are often of no-Fog LVP type during the day time hours and are associated with the scenario where the decision height decreases below $60 \mathrm{~m}$ (HCB-LVP) and they never occur when RVR is below 600m (RVR-LVP) during the daylights.

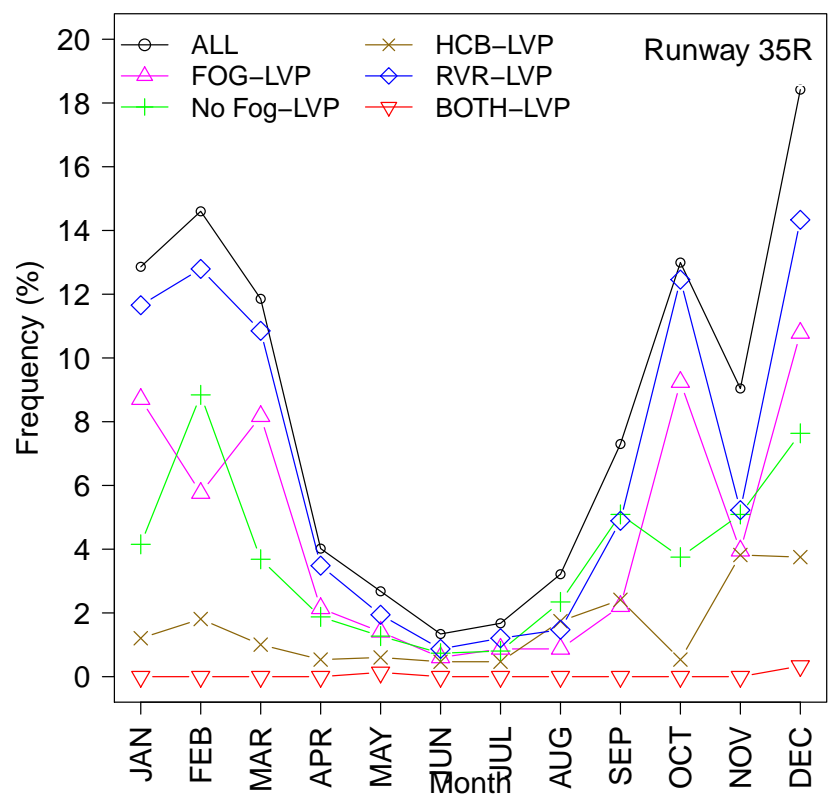

(a)

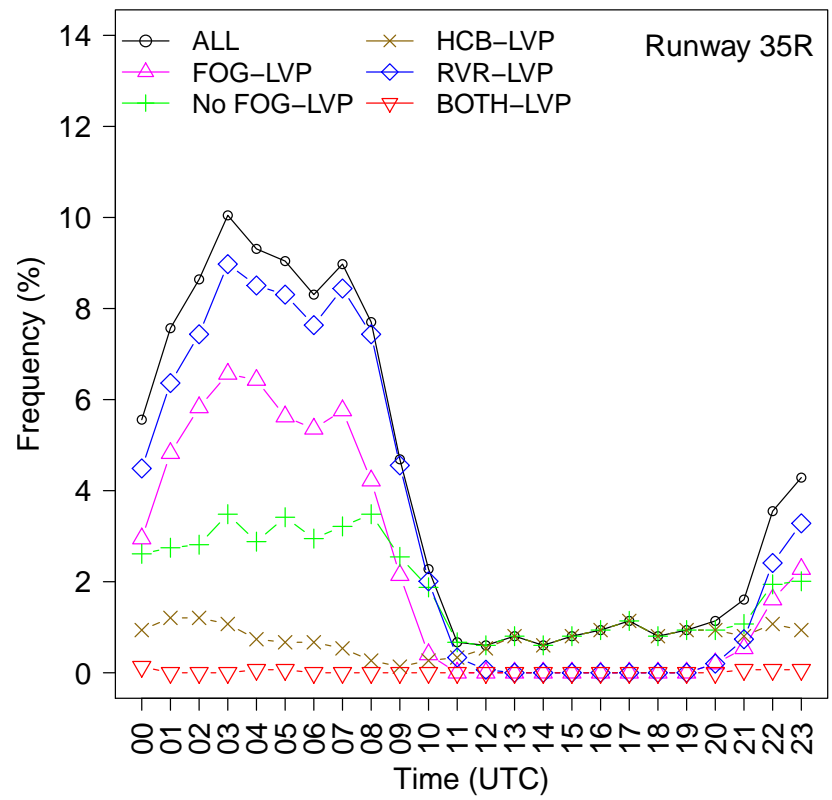

(c)

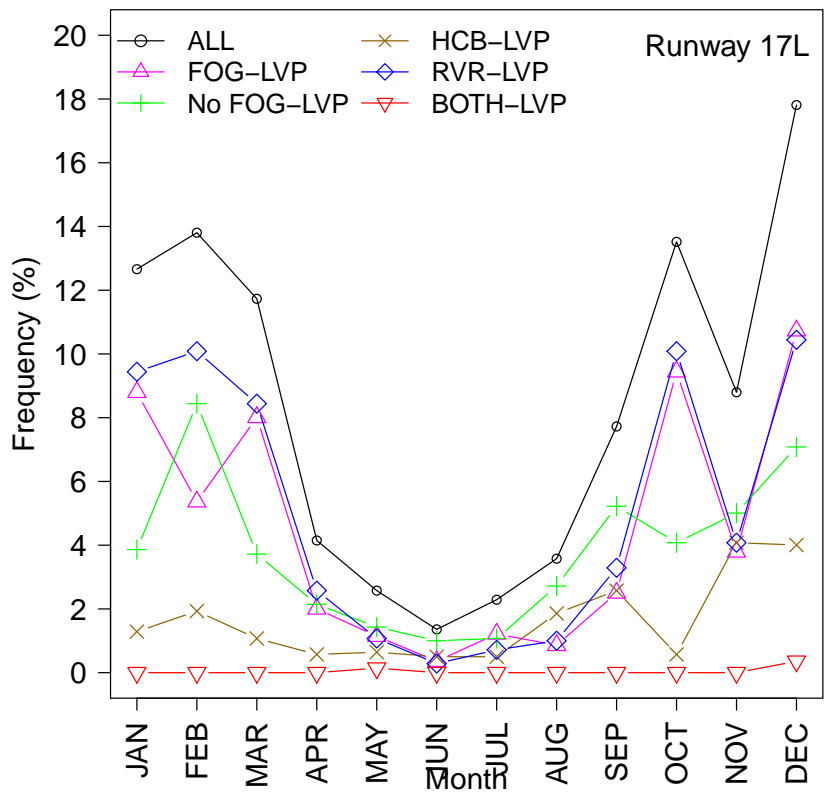

(b)

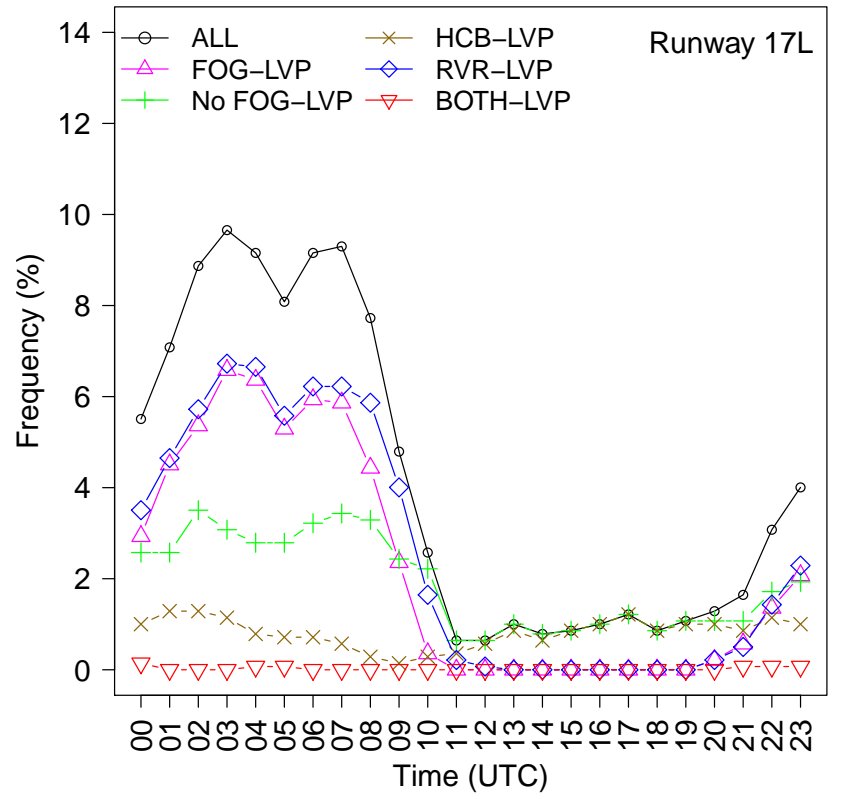

(d)

Figure 1: (top) Monthly and (bottom) hourly distribution of LVP conditions' occurrence at runway 35R (left) and 17L (right). The dataset covers the entire period from January 2009 to March 2015. 


\subsection{Numerical results of Neural Networks}

The performance of the developed models was assessed using the three aforementioned measures (POD, FAR and FBI). Fig. 2 displays the verification scores (Fig. 2(a) and 2(d) for POD, Fig. 2(b) and 2(e) for FAR, and Fig. $2(\mathrm{c})$ and $2(\mathrm{f})$ for FBI) of each developed ANN model as function of valid forecast time (UTC) for each runway end point (35R and 17L). This figure shows that most of the ANNs have a probability of detection higher than $70 \%$ and reaches $100 \%$ for some models. The false alarm rate does not exceed $50 \%$, particularly for valid forecast upon 2300UTC. The frequency bias index (FBI) of the all the models fits well and still around 1.5 (the perfect value for this score is 1) expect for runway 17L where some models have a poor bias due to the fact that these models do not forecast the LVP conditions for some valid forecast time (Fig. 2(f)). This could be due to the high fluctuations in instantaneous measurements from AWOS used in this work or to the sensitivity of the instruments. This will be discussed later. But, in a general sense, most of the developed models are able to well predict LVP conditions occurring during the night and early morning, particularly for the runway $35 \mathrm{R}$.

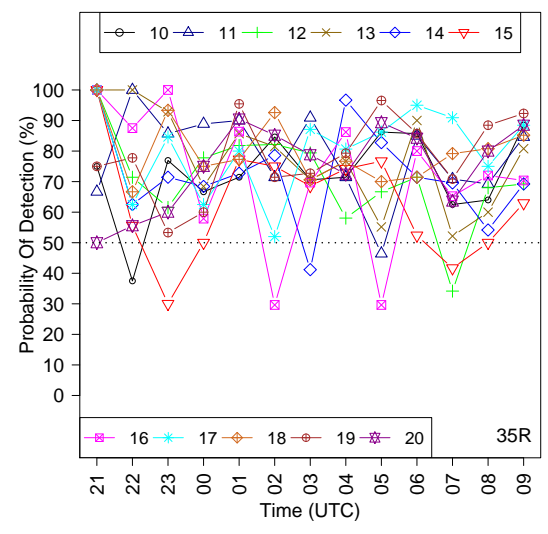

(a)

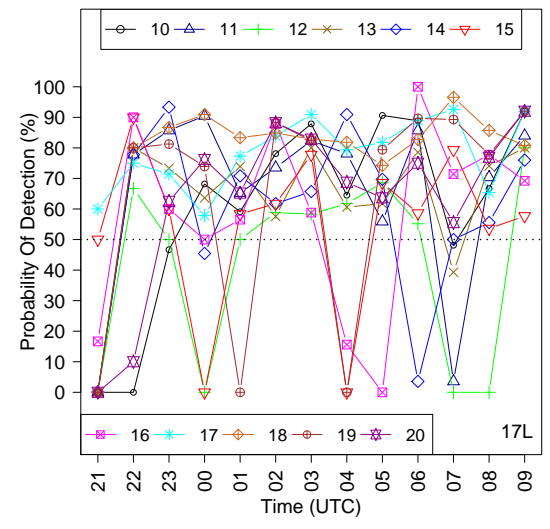

(d)

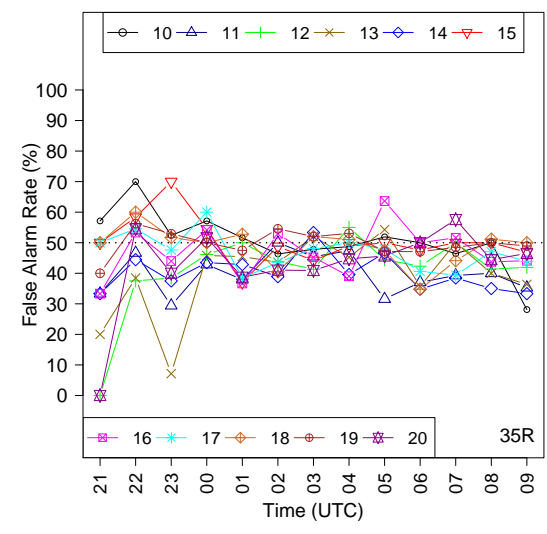

(b)

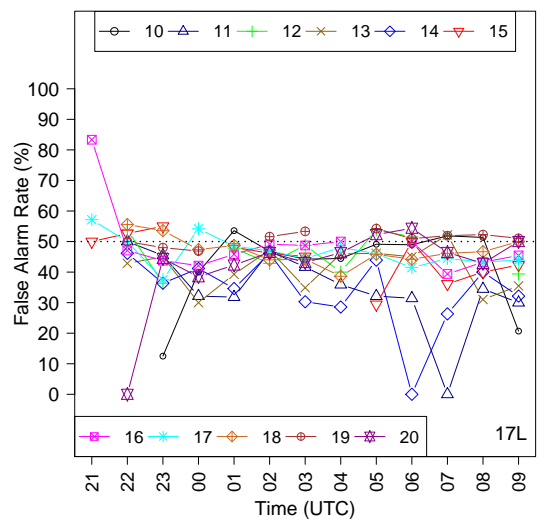

(e)

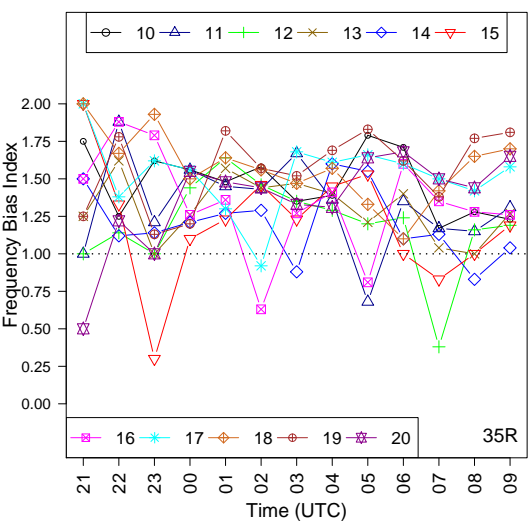

(c)

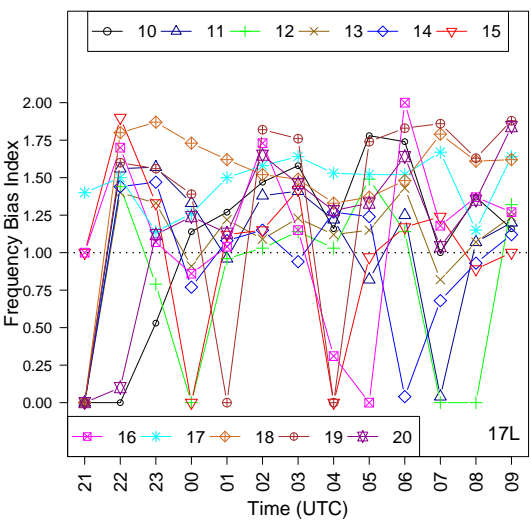

(f)

Figure 2: Hourly distribution of the verification scores as function of the valid times for each ANN-based model for (top) runway 35R and (bottom) runway 17L: (a) and (d) Probability of detection (POD), (b) and (e) False alarm rate (FAR), (c) and (f) frequency bias index (FBI).

Operationally, many random errors can affect meteorological observations. This could be due to the sensitivity of the instruments. Thus, a sensitivity experiment is carried out to assess the robustness of the ANN-based developed models to errors or fluctuations in input parameters. This will be discussed in section 3.2.1. Besides, the correlation between the rate of false alarms and the LVP type and source is examined in section 3.2.2. 


\subsubsection{Sensitivity to errors in input parameters}

In order to assess the ANN's ability to cope with errors in input parameters, new testing subsets were created where the observations were randomly perturbed with errors of fixed magnitude. Table 1 summarizes the magnitude of perturbation added to the observed values in the new testing subsets.

Table 1: The magnitude of perturbation added randomly to the observed values in order to cerate the new testing subsets.

\begin{tabular}{lc}
\hline Parameter & Perturbation magnitude \\
\hline Temperature $\left({ }^{\circ} \mathrm{C}\right)$ & \pm 0.5 \\
Pressure $(\mathrm{hPa})$ & \pm 1 \\
Wind speed $(\mathrm{m} / \mathrm{s})$ & \pm 1 \\
Wind direction (degrees) & \pm 10 \\
Relative Humidity $(\%)$ & \pm 5 \\
Runway Visual Range $(\mathrm{m})$ & \pm 10 \\
Cloud base height $(\mathrm{m})$ & \pm 10 \\
\hline
\end{tabular}

According to the magnitude of perturbations mentioned in Table 1 , the relative error $(R E)$ was from $1 \%$ to $10 \%$ of the observed values where the relative error is defined by:

$R E=\frac{x_{\text {perturb }}-x_{\text {true }}}{x_{\text {true }}} * 100$

where $x_{\text {true }}$ and $x_{\text {perturb }}$ refer respectively to the actual observed value and the perturbed one.

Table 2 summarizes the mean and standard deviation for the difference of verification scores $(P O D, F A R$ and $F B I$ ) between the reference experiment and the perturbed one $\left(S_{\text {core }}\right.$ ref $-S_{\text {core }}$ perturb $)$ for both runway end points $35 \mathrm{R}$ and $17 \mathrm{~L}$. This table covers all the lead and valid times. It shows that the ANN-based developed models are robust to low level relative errors in input parameters for runway 35R while the POD differences have a high dispersion around their mean value for runway 17L. In fact, it is found that the mean value of the difference for the probability of detection is about $-0.41 \%$ for runway $35 \mathrm{R}$ and $4.83 \%$ for runway $17 \mathrm{~L}$. The mean value of the false alarm rate is of about $-0.21 \%$ for runway $35 \mathrm{R}$ while these scores are similar in average between the two experiments for runway 17L. It should be noticed that there is a big difference between the standard deviation for the two runway end points (15.09 vs 22.48 for POD and 10.09 vs 7.24 for FAR). Comparing the two experiments, the frequency bias index are similar (mean value of 0.0 for $35 \mathrm{R}$ and 0.9 for $17 \mathrm{~L}$ associated with a weak standard deviation of 0.2 for $35 \mathrm{R}$ and 0.44 for $17 \mathrm{~L})$.

Table 2: Mean and standard deviation for the difference between the verification scores for the perturbed experiment and the reference one. The statistic covers all the lead and valid times.

\begin{tabular}{l|cc|cc}
\hline \multirow{2}{*}{ Verification Score } & \multicolumn{2}{|c|}{ Runway 35R } & \multicolumn{2}{c}{ Runway 17L } \\
\cline { 2 - 5 } & Mean & Standard Deviation & Mean & Standard Deviation \\
\hline POD & -0.41 & 15.09 & 4.83 & 22.48 \\
FAR & -0.21 & 7.24 & 0.00 & 10.09 \\
FBI & 0.00 & 0.28 & 0.09 & 0.44 \\
\hline
\end{tabular}

\subsubsection{LVP type and source oriented analysis}

Successful LVP conditions' prediction depends on the LVP type that is being predicted. The LVP conditions were classified into FOG LVP and no-FOG LVP categories. Besides, the LVP sources were fixed in three classes: RVRLVP, HCB-LVP and both of them. Thus, to deeply investigate the correlation between the rate of false alarms and the LVP type and source occurrence at the Mohamed V airport, the rate of missed LVP hours as function of the LVP type, issued from the classification previously described, is summarized in Table 3. For each LVP type and source, the percentage of missed LVP hours is the ratio between their number of occurrence and the total number of LVP hours. 
Table 3: Total number of LVP hours, numbers of well predicted and missed LVP hours, and the percentage of missed LVP hours as function of the LVP types (Fog LVP and no-Fog LVP) and of LVP source (RVR, HCB or both). The statistic covers all the testing samples used in this study.

\begin{tabular}{l|ll|lll|ll|lll}
\hline & \multicolumn{4}{|c|}{ Runway 35R } & \multicolumn{4}{c}{ Runway 17L } \\
\cline { 2 - 10 } & Fog & No-Fog & RVR & HCB & Both & Fog & No-Fog & RVR & HCB & Both \\
\hline Total number of LVP hours & 2083 & 720 & 2794 & 9 & 0 & 2609 & 881 & 3481 & 9 & 0 \\
Total number of well predicted LVP hours & 1544 & 532 & 2069 & 7 & 0 & 1691 & 608 & 2297 & 2 & 0 \\
Total number of missed LVP hours & 539 & 188 & 725 & 2 & 0 & 918 & 273 & 1184 & 7 & 0 \\
Percentage of missed LVP hours (\%) & 25.88 & 26.11 & 25.95 & 22.22 & 0 & 35.19 & 30.99 & 34.01 & 77.78 & 0 \\
\hline
\end{tabular}

Table 3 points out that the developed ANN-models are not clearly sensitive to a specific LVP type and source for runway 35R (percentage of missed LVP hours does not exceed 26\%) while for runway 17L, the percentage of missed LVP hours is slightly high (more than 30\%), in particular for HCB-LVP where the percentage reaches $77.78 \%$. This confirms the prevalence of ANN-models skill for 35R in comparison of those of 17L.

\section{Conclusion}

In this paper, the local characteristics of low visibility and/or ceiling (LVP conditions) at the Mohamed V international airport, Morocco, has been presented using the meteorological parameters from the Automated Weather Observation Station (AWOS) over the period from January 2009 to March 2015. Results have shown that most of LVP conditions are associated with fog and are often due to decreasing of runway visual range below the LVP threshold $(600 \mathrm{~m})$. It is also found that LVP conditions mostly occur during the cold season (from October to April), particularly in winter months and they are often observed during the night time and early morning hours (from 2100UTC to 0900UTC). In attempt to provide Air Traffic Control authorities with accurate forecasts which include the probability of visibility and/or ceiling conditions which may require LVP to be undertaken, a set of ANN-based models have been developed using the resilient backpropagation as supervised learning algorithm. It is found that these models have good LVP prediction ability during night-time at Mohamed V international airport. Besides, sensitivity experiment to errors in input parameters points out the robustness of the developed models, particularly for runway 35R. Furthermore, investigation in the correlation between the LVP type that is being predicted and the rate of false alarm demonstrates that the ANNs are slightly sensitive to the LVP type that occurred at the airport. Such developed ANNs can be helpful for operational weather forecasters. Then, ANN-based models can similarly be developed for other Moroccan LVP-prone airports like Kenitra and Rabat.

\section{Acknowledgements}

Dr Mohamed Nabhani from DMN is acknowledged for the fruitful discussions during this study and for his valuable comments.

\section{References}

[1] Bergot, T., "Quality assessment of the Cobel-Isba numerical forecast system of fog and low clouds". Pure and Applied Geophysics, Vol. 164, No. 6-7, (2007), 1265-1282.

[2] ICAO, "European guidance material on All weather operations at aerodromes", European and North Atlantic office, Fourth edition (2012). "available online: http://www.icao.int/EURNAT/"

[3] WMO, "International Meteorological Vocabulary", WMO: Geneva, Switzerland, (1992).

[4] M.W. Gardner, S.R. Dorling, "Artificial neural networks (the multilayer perceptron)- A review of applications in the atmospheric sciences", Atmospheric environment, Vol. 32, (1998), 2627-2636.

[5] W.W. Hsieh, B. Tang, "Applying neural network models to prediction and data analysis in meteorology and oceanography", Bulletin of the American Meteorological Society, Vol. 79, (1998), 1855-1870.

[6] A. Pasini, V. Pelino, S. Potesta, "A neural network model for visibility nowcasting from surface observations: Results and sensitivity to physical input variables", Journal of Geophysical Research, Vol. 106, (2001), 14951-14959. 
[7] D. Fabian, R. De Dear, S. Lellyeit, "Application of artificial neural network forecasts to predict fog at Canberra international airport", Weather and Forecasting, Vol. 22, (2006), 372-381.

[8] J.B. Bremnes, S.C. Michaelides, "Probabilistic visibility forecasting using neural networks", Pure and Applied Geophysics, Vol. 164, (2007), 1365-1381.

[9] C. Marzban, S.M Leyton, B. Colman, "Ceiling and visibility forecasting via neural networks", Weather and Forecasting, Vol. 22, (2006), 466-479.

[10] F. Günther, S. Fritsch, "Neuralnet : Training of Neural Networks", The R Journal, Vol. 2, (2010), 30-38.

[11] G. Zhang, B. Eddy Patuwo, M.Y. Hu, "Forecasting with artificial neural networks : the state of the art", International Journal of Forecasting, Vol. 14, (1998), 35-62.

[12] D.E. Rumelhart, J.L. Mc Clellend, "Parallel distributed Processing : Explorations in the microstructure of cognition", MIT Press, Cambridge, (1986), 318-362.

[13] A.D. Anastasiadis, G.D. Magoulas, M.N. Vrahatis, "New globally convergent training scheme based on the resilient propagation algorithm", Neurocomputing, Vol. 64, (2005), 253-270.

[14] S. Knerr, L. Personnaz, G. Dreyfus, "Single-layer learning revisited: a stepwise procedure for building and training a neural network". In Neurocomputing. Springer Berlin Heidelberg. (1990), 41-50.

[15] C.R. Parikh, M.J. Pont, N.B. Jones, "Improving the performance of multi-layer perceptrons where limited training data are available for some classes". Proc. Ninth Int. Conf. on Artificial Neural Networks, Edinburgh, United Kingdom, Institution of Electrical Engineers. Vol. 1, (1999), 227-232.

[16] D.B. Reusch, R.B. Alley. "Automatic weather stations and artificial neural networks: Improving the instrumental record in West Antarctica". Mon. Wea. Rev., Vol. 130, (2002), 3037-3053.

[17] D.S. Wilks, "Statistical Methods in the Atmospheric Sciences". Academic Press, (1995), 467pp. 